\title{
Vacuum polarization effects on quasinormal modes in electrically charged black hole spacetimes.
}

\author{
Jeferson de Oliveira* \\ Institute of Physics, University of São Paulo, Brazil \\ E-mail: jefersonefma.if.usp.br
}

\section{Owen Pavel Fernandez Piedra}

Departamento de Física y Química, Facultad de Mecánia, Universidad de Cienfuegos, Cuba

E-mail: opaveleucf.edu.cu

\begin{abstract}
We investigate the influence of vacuum polarization of quantum massive fields on the scalar sector of quasinormal modes in spherically symmetric black holes. We consider the evolution of a massless scalar field on the spacetime corresponding to a charged semiclassical black hole, consisting of the quantum corrected geometry of a Reissner-Nordström black hole dressed by a quantum massive scalar field in the large mass limit. Using a sixth order WKB approach we find a shift in the quasinormal mode frequencies due to vacuum polarization .
\end{abstract}

5th International School on Field Theory and Gravitation, April 20 - 242009

Cuiabá city, Brazil

*Poster Session 


\section{Introduction}

The evolution of a small perturbation in a black hole background geometry gives rise, under appropriate boundary conditions to a discrete set of complex frequencies called quasinormal frequencies. Studying the quasinormal spectrum of black holes we can gain some valuable information about these objects, since quasinormal evolution depends only upon the parameters of black hole itself. Thus these frequencies represents the caracteristic resonance spectrum of a black hole response [1]. In addition, we can investigate the black holes stability against small perturbations. Further contexts include astrophysical black holes [1] [2] and the AdS/CFT correspondence, where the inverse of imaginary part of quasinormal frequencies of AdS black holes can be interpreted as the dual CFT relaxation time [3].

An interesting problem consists in determining what changes appears in the quasinormal mode spectrum of a black hole if we consider such a system surrounded by a quantum field with a semiclassical gravity, leading to a quantum corrected line element for the dressed black hole. A similar work was done by Konoplya [4], for the BTZ black hole dressed by a massless scalar field, in which the particle creation around the event horizon dominates over the vacuum polarization effect. This paper is devoted to find this vacuum polarization effects upon quasinormal modes of quantum corrected Reissner-Nordström black holes in four dimensions. In the following we use for the Riemann tensor, its contractions, and the covariant derivatives the sign conventions of Misner, Thorne and Wheeler [5]. Our units are such that $\hbar=c=G=1$.

\section{Renormalized stress energy tensor for quantum scalar massive field}

Consider a single quantum scalar field $\phi(x)$ with mass $m$ interacting with gravity with non minimal coupling constant $\xi$ in four dimensions. In the large mass limit the one loop effective action for the quantized scalar field $[6,7,8,9]$ is given by

$$
W_{\text {ren }}^{(1-\text { loop })}=\frac{1}{6(4 \pi)^{2} m^{2}} \int d^{4} x \sqrt{-g} \operatorname{stra}_{3}(x, x),
$$

where strF denotes the functional supertrace of $F$, and $a_{3}(x, x)$ is the coincidence limit of fourth Hadamard-Minakshisundaram-DeWitt-Seeley coefficient (HMDS). Upon inserting the expression for the HMDS coefficient in the above formula for the effective action we obtain a renormalized effective lagrangian $[7,8,9,10]$ given by $\mathfrak{L}_{\text {ren }}=\mathfrak{L}_{\text {ren }}^{\text {conformal }}+\widetilde{\mathfrak{L}_{\text {ren }}}$, where the conformal part of the effective lagrangian is

$$
\begin{aligned}
\mathfrak{L}_{\text {ren }}^{\text {conformal }} & =\frac{1}{192 \pi^{2} m^{2}}\left[\frac{1}{7560} \Theta R \square R+\frac{1}{140} R_{\mu v} \square R^{\mu v}-\frac{8}{945} R_{v}^{\mu} R_{\gamma}^{v} R_{\mu}^{\gamma}+\frac{2}{315} R^{\mu v} R_{\gamma \rho} R_{\mu \nu}^{\gamma \rho}\right. \\
& \left.+\frac{1}{1260} R_{\mu \nu} R_{\sigma \gamma \rho}^{\mu} R^{v \sigma \gamma \rho}+\frac{17}{7560} R_{\gamma \rho}{ }^{\mu v} R_{\mu \nu}{ }^{\sigma \tau} R_{\sigma \tau}{ }^{\gamma \rho}-\frac{1}{270} R_{\mu \nu}^{\gamma \rho} R_{\sigma \tau}^{\mu \nu} R_{\gamma \rho}^{\sigma \tau}\right]
\end{aligned}
$$

and the mass dependent contribution takes the form

$$
\widetilde{\mathfrak{L}_{r e n}}=\frac{1}{192 \pi^{2} m^{2}}\left[\frac{1}{30} \eta\left(R R_{\mu v} R^{\mu \nu}-R R_{\mu v \gamma \rho} R^{\mu v \gamma \rho}\right)+\frac{1}{2} \eta^{2} R \square R-\eta^{3} R^{3}\right],
$$


where we use $\Theta=30-252 \xi$ and $\eta=\xi-\frac{1}{6}$. By standard functional differentiation of the effective action with respect to the metric, the renormalized Stress-Energy tensor is obtained being given by $\left\langle T_{\mu v}\right\rangle_{\text {ren }}=\frac{2}{\sqrt{-g}} \frac{\delta W_{\text {ren }}}{\delta g^{\mu \nu}}=C_{\mu}{ }^{v}+D_{\mu}{ }^{v}$, where the $C_{\mu}{ }^{v}$ and $D_{\mu}{ }^{v}$ tensors take cumbersome forms that the reader can find in $[9,10]$. For the present work we deal with the Reissner-Nordström spacetime. This results can be found in the paper by Matyjasek [8] and is amazingly simple, $\left\langle T_{v}^{\mu}\right\rangle=C_{v}^{\mu}+\left(\xi-\frac{1}{6}\right) D_{v}^{\mu}$.

These results were obtained using the expression for the stress energy tensor presented in [9] and coincides with that previously obtained by Matyjasek in [8] using also the SchwingerDeWitt approximation. In our chosen system of units the general condition for the validity of the Schwinger-DeWitt approximation can be put as $m M \geq 1$, where $m$ and $M$ are respectively the quantum scalar field and black hole masses.

\section{Semiclassical solution}

In this section we show how to solve the general backreaction problem for spherically charged symmetric spacetimes. We will follow the lines of reference [11].

Consider the line element for a general spherically symmetric spacetime

$$
d s^{2}=-A(r) d t^{2}+B(r) d r^{2}+r^{2}\left(d \theta^{2}+\sin ^{2} \theta d \phi^{2}\right) .
$$

We intend to solve the general semiclassical Einstein equations with the source $T_{\mu \nu}=T_{\mu \nu}^{\text {class }}+$ $\left\langle T_{\mu \nu}\right\rangle$ including two contributions: the first, denoted by $T_{\mu \nu}^{\text {class }}$, comes from a classical source, and the second, denoted by $\left\langle T_{\mu v}\right\rangle$, is the quantum field contribution. It is possible to show, using appropiate combinations of the components of the Ricci tensor for the line element (3.1), that the general form for the metric components $g_{r r}=B(r)$ and $-g_{t t}=A(r)$ that solves the backreaction problem are given by $\frac{1}{B(r)}=1-\frac{2 M}{r}+\frac{Q^{2}}{r^{2}}+\frac{8 \pi}{r} \int_{\infty}^{r} \zeta^{2}\left\langle T_{t}^{t}\right\rangle d \zeta$, and $A(r)=\frac{1}{B(r)} \exp \{\lambda(r)\}$, where $\lambda(r)=8 \pi \int_{\infty}^{r} \zeta B(\zeta)\left(\left\langle T_{r}^{r}\right\rangle-\left\langle T_{t}^{t}\right\rangle\right) d \zeta$. In this equations $Q$ and $M$ denotes the charge and the bare mass of the black hole, i.e, of the classical Reissner-Nördström solution. Note that the general solutions above are obtained using the same boundary conditions of reference [11]. Usually, the integrals in $1 / B(r)$ and $\lambda(r)$ are performed introducing some perturbation approximation due to the fact that the quantum stress tensor $\left\langle T_{\mu \nu}\right\rangle$ is linear in the Dirac constant $\hbar$ ( that in our chosen units is $\hbar=1$ ). We use as a perturbation parameter the ratio $\varepsilon=1 / M^{2}$, where $\mathrm{M}$ is the mass of the black hole ( in conventional units we have $\varepsilon=M_{P}^{2} / M^{2}$, where $M_{P}$ is the Planck's mass). After some algebra we obtain $\frac{1}{B(r)}=1-\frac{2 M}{r}+\frac{Q^{2}}{r^{2}}+\frac{\varepsilon}{\pi m^{2}}(F(r)+\xi H(r))$, where, the explicit form of the functions $F(r), H(r)$ and $\lambda(r)$ can be found in [12]

With these analytical results for the semiclassical line element representing a quantum corrected charged black hole, we can determine the changes in some of the properties of the solution, with respect to its classical counterpart. In particular, we can determine the change in the position of the event horizon due to quantum effects. Let be $r_{h}=M+\sqrt{M^{2}-Q^{2}}$ the position of the event horizon for the classical charged black hole. The exact horizon position $r_{+}$for the semiclassical charged black hole is given by $r_{+}=r_{h}\left(1-\varepsilon\left(\frac{4 \pi}{\left(M-Q^{2} / r_{h}\right)} \int_{\infty}^{r_{h}} \zeta^{2}\left\langle T_{t}^{t}(\zeta)\right\rangle\right) d \zeta\right)$. Now using the expressions for the temporal component of the stress-energy tensor of the quantum field, and performing the above integral, we obtain the result for the position of event horizon. It is interesting 
to note that the effect of the quantum field over the bare black hole spacetime is to reduce the position of the event horizon. This reduction is a consequence of the typical fact that the weak energy condition for the quantum field is violated on the event horizon.

\section{Looking for scalar quasinormal frequencies}

In this section, we consider the evolution of a test massless scalar field $\Phi\left(x^{\mu}\right)$, where $x^{\mu}=$ $(t, r, \theta, \phi)$, in the background of the semiclassical spherically charged black hole studied above. The dynamics of for this test field is governed by the Klein-Gordon equation $\square \Phi=0$. Changing the wave function $\Phi=\Psi / r$, and the radial coordinate $d r / d r_{*}=\sqrt{B(r) / A(r)}$, and separating the time, radial and angular dependence of the field as $\Psi=e^{i \omega t} Z(r)_{L} Y_{L m}(\theta, \phi)$, the Klein-Gordon equation is written as $\frac{d^{2}}{d r_{*}^{2}} Z_{L}-\left[\omega^{2}-V\right] Z_{L}=0$, where $\omega$ is the quasinormal frequency and $V$ is the effective potential. The potential $V$ is a function of the metric components $g_{\mu \nu}$ and the multipolar number $L$. For the specific case discussed in this paper, we have for the effective potential the general result $V(r)=V^{c}(r)+\frac{\varepsilon}{\pi m^{2}} U(r)+O\left(\varepsilon^{2}\right)$, where $V^{c}(r)$ is the scalar effective potential of the bare Reissner-Nordstrom black hole given by $V^{c}(r)=\left(r^{2}-2 M r+Q^{2}\right)\left(-2 Q^{2}+\beta r^{2}+2 M r\right) r^{-6}$, and $U(r)$ is proportional to the first order contribution of the vacuum polarization effect to the total effective potential. Again, the explicit expressions can be found in the full version of the present work [12].

The effective potential $V$ has the form of a definite positive potential barrier, i.e, it is a well behaved function that goes to zero at spatial infinity and gets a maximum value near the event horizon. The quasinormal modes are solutions of the above wave equation with the specific boundary conditions requiring pure out-going waves at spatial infinity and pure in-coming waves on the event horizon. In order to evaluate the quasinormal modes for the test scalar field, we use the well known WKB technique, that can give accurate values of the lowest ( that is longer lived ) quasinormal frequencies. We use in our numerical calculation of quasinormal modes this sixth order WKB expansion, for which was shown in [13] that gives a relative error which is about two order less than the third WKB order. In table (1) we list values for the real and imaginary parts of the quasinormal frequencies for semiclassical and classical black holes varying the multipolar number $L$ and the overtone number.

As the obtained numerical results show a shift on the quasinormal spectrum due to semiclassical corrections on the Reissner-Nordström background appears, an effect that is more pronounced for the fundamental mode $(L=0)$. From (1) and (2) we see that the backreaction of the quantized field upon the classical solution gives rise to a decreasing of the real oscillation frequencies and to a small decreasing of the damping rate, for physically interesting values of the black hole mass.

We also evaluate the dependence of the quasinormal frequencies for a given black hole bare mass and different values of the coupling constant between the quantum field of fixed mass $m$ and the classical background spacetime including the more physically interesting cases of minimal and conformal coupling. The results appears in figure (3) and (4).

\section{Concluding remarks}

We have studied the influence of the backreaction due to vacuum polarization on the structure 


\begin{tabular}{|c|c|c|c|c|c|c|c|}
\hline \multicolumn{4}{|c|}{ Semiclassical solution } & \multicolumn{4}{|c|}{ Classical solution } \\
\hline \multicolumn{8}{|c|}{$M=100$} \\
\hline$L$ & $n$ & $\operatorname{Re}(\varpi)$ & $-\operatorname{Im}(\varpi)$ & $L$ & $n$ & $\operatorname{Re}(\varpi)$ & $-\operatorname{Im}(\varpi)$ \\
\hline 0 & 0 & 2.3302 & 1.2140 & 0 & 0 & 14.233 & 1.0638 \\
\hline 1 & 0 & 3.5226 & 1.1772 & 1 & 0 & 3.8461 & 1.1606 \\
\hline 1 & 1 & 3.1836 & 3.6897 & 1 & 1 & 8.1164 & 2.4344 \\
\hline \multicolumn{8}{|c|}{$M=110$} \\
\hline$L$ & $n$ & $\operatorname{Re}(\varpi)$ & $-\operatorname{Im}(\varpi)$ & $L$ & $n$ & $\operatorname{Re}(\varpi)$ & $-\operatorname{Im}(\varpi)$ \\
\hline 0 & 0 & 1.2090 & 1.1042 & 0 & 0 & 6.0223 & 0.9657 \\
\hline 1 & 0 & 3.2054 & 1.0698 & 1 & 0 & 3.4741 & 0.9579 \\
\hline 1 & 1 & 2.8942 & 3.3544 & 1 & 1 & 5.1130 & 1.5760 \\
\hline \multicolumn{8}{|c|}{$M=150$} \\
\hline$L$ & $n$ & $\operatorname{Re}(\varpi)$ & $-\operatorname{Im}(\varpi)$ & $L$ & $n$ & $\operatorname{Re}(\varpi)$ & $-\operatorname{Im}(\varpi)$ \\
\hline 0 & 0 & 1.0230 & 0.9337 & 0 & 0 & 2.1751 & 0.8333 \\
\hline 1 & 0 & 2.7123 & 0.9053 & 1 & 0 & 2.9588 & 0.8928 \\
\hline 1 & 1 & 2.4490 & 2.8383 & 1 & 1 & 6.2429 & 1.8724 \\
\hline
\end{tabular}

Table 1: Rescaled Scalar quasinormal frequencies $\varpi=10^{3} \omega$ for the classical and semiclassical ReissnerNordström black hole, with $Q / M=0.75, m=1 / 10$ and $\xi=0$.

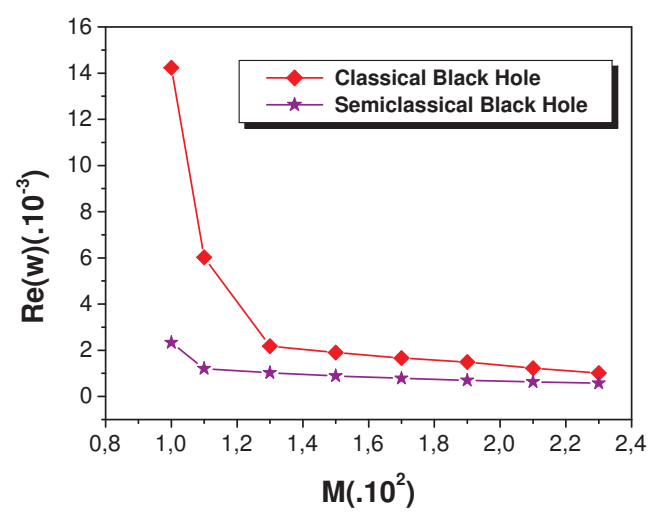

Figure 1: $\operatorname{Re}(\omega)$ for classical and semiclassical black holes.

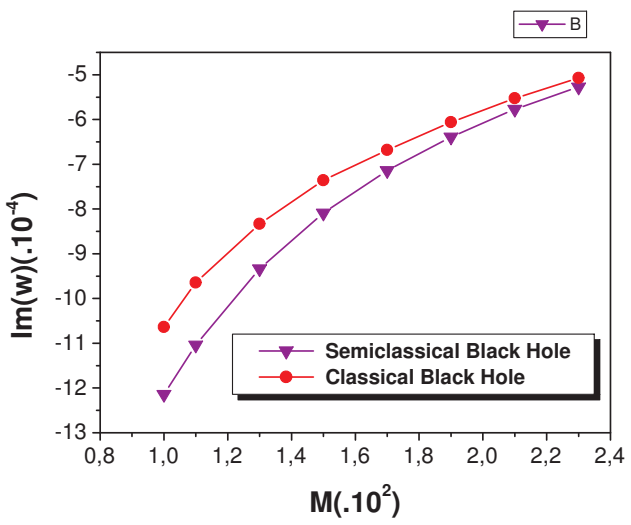

Figure 2: $\operatorname{Im}(\omega)$ for classical and semiclassical black holes.

of scalar quasinormal frequencies for semiclassical charged black holes. Such an influence appears essentially as an appreciable shift in the quasinormal frequencies that decreases as the bare black hole mass increases, and that not have a strong dependance upon the quantum field parameters. This shift shows that the quantum corrected quasinormal modes are less oscillatory with respect to its classical counterpart.

\section{Acknowledgments}

This work has been supported by FAPESP and CNPQ, Brazil as well as ICTP, Trieste. 


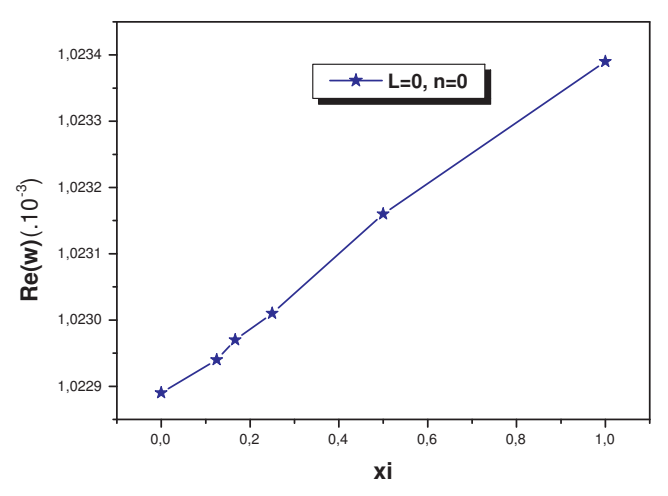

Figure 3: Behaviour of the $\operatorname{Re}(\omega)$ for different values of couplig constant $\xi$.

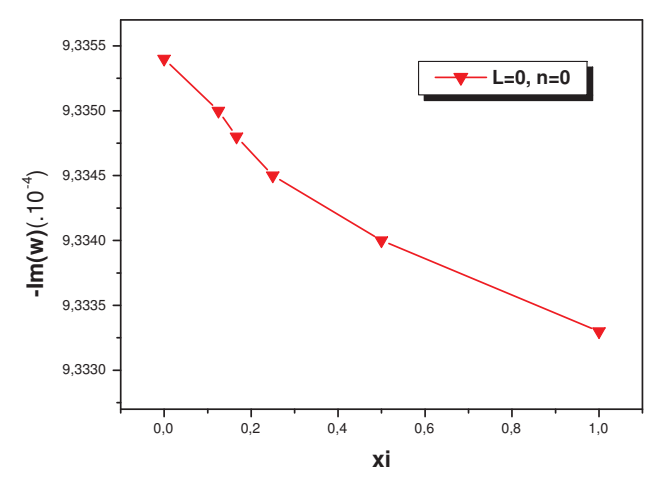

Figure 4: Behaviour of the $\operatorname{Im}(\omega)$ for different values of couplig constant $\xi$.

\section{References}

[1] K. D. Kokkotas and B. G. Schmidt, Quasi-normal modes of stars and black holes, Living Rev. Rel. 2, 2 (1999) [arXiv:gr-qc/9909058].

[2] H. P. Nollert, Quasinormal modes: the characteristic 'sound' of black holes and neutron stars, Class. Quant. Grav. 16, R159 (1999).

[3] G. T. Horowitz and V. E. Hubeny, Quasinormal modes of AdS black holes and the approach to thermal equilibrium, Phys. Rev. D 62, 024027 (2000) [arXiv:hep-th/9909056].

[4] R. A. Konoplya, Influence of the back reaction of the Hawking radiation upon black hole quasinormal modes, Phys. Rev. D 70, 047503 (2004) [arXiv:hep-th/0406100].

[5] C. W. Misner, K. S. Thorne and J. A. Wheeler, Gravitation, (Freeman, San Francisco, 1973).

[6] V. P. Frolov and A. I. Zelnikov, Vacuum Polarization By A Massive Scalar Field In Schwarzschild Space-Time, Phys. Lett. B 115, 372 (1982).

[7] I. G. Avramidi, A covariant technique for the calculation of the one-loop effective action, Nucl. Phys. B 355, 712 (1991) [Erratum-ibid. B 509, 557 (1998)].

[8] J. Matyjasek, Stress-energy tensor of neutral massive fields in the Reissner-Nordstroem spacetime, Phys. Rev. D 61, 124019 (2000) [arXiv:gr-qc/9912020].

[9] O. P. Fernandez Piedra and A. C. Montes de Oca, Quantization of massive scalar fields over static black string backgrounds, Phys. Rev. D 75, 107501 (2007) [arXiv:0707.0706 [gr-qc]].

[10] O. P. Fernandez Piedra and A. C. M. de Oca, Quantization of massive scalar fields over axis symmetric space-time backgrounds, arXiv:gr-qc/0701135.

[11] C. O. Lousto and N. G. Sanchez, Back reaction effects in black hole space-times, Phys. Lett. B 212 , 411 (1988).

[12] O. P. F. Piedra and J. de Oliveira, Vacuum polarization effects on quasinormal modes in electrically charged black hole spacetimes, arXiv:0902.1487 [gr-qc].

[13] R. A. Konoplya, Quasinormal modes of the Schwarzschild black hole and higher order WKB approach, J. Phys. Stud. 8, 93 (2004). 